\title{
QUALCHE CONSIDERAZIONE SUL "RETOROMANZO"
}

Nella complessa discussione circa la posizione linguistica del "retoromanzo" (o "ladino", in senso ampio), una entità dialettologica ritenuta autonoma e per lo più unitaria in seno alle lingue neolatine secondo le trattazioni di tutti (o quasi tutti) $i$ manuali di linguistica romanza, sarà opportuno riesaminare brevemente la concezione di G.I. Ascoli che deve essere considerato il vero iniziatore di tale dibattito, condotto con notevole ricchezza di materiali (per i suoi tempi) e con un metodo scientifico. Le osservazioni degli storici, di altri studiosi e i brevi cenni dei linguisti prima della pubblicazione dei "Saggi ladini“, del 1873, sono infatti in comparazione, molto approssimative (più spesso errate) e ben poca cosa. ${ }^{1}$

Qui non ci soffermeremo a considerare nuovamente la posizione di TH. Gartner e di altri glottologi, venuti dopo, i quali hanno anche tentato di giustificare storicamente la teoria del "retoromanzo" che è considerata per lo più inoppugnabile e da tempo scontata, divulgata anche a livello popolare ed in generale assai gradita ai parlanti locali. Questo mio breve intervento costituisce una delle sintetiche premesse ad un contributo ben più ampio e documentato che sto preparando per la massima rivista di romanistica ove, mi auguro, sarà, tra non molto, ospitato.

Come è ben risaputo, è stato soprattutto l'Ascoli a diffondere nella terminologia scientifica delle lingue neolatine, la denominazione di "ladino" per indicare il "gruppo di favelle" che egli riteneva assai affini tra di loro tanto da costituire un gruppo dialettale autonomo, pare differenziato dal "cisalpino" o italiano settentrionale (ma qui la sua formulazione risulta francamente confusa!). Egli optò per il termine suddetto verosimilmente perché "ladino" (da la t in u s) era denominazione indigena e popolare nella Val Badia, ove una parte della popolazione (circa 1900 abitanti secondo la stima del Gartner $1883, \mathrm{XX}$ ) del distretto o circondario di Wengen, cioè La Valle, Campill e San Martino della Torre sulla sinistra del Gàdera come contrapposizione agli altri valligiani dipendenti da Castel Badia (Sonnenburg) che si denominavano badiòc'. La denominazione era inoltre nota anche per indicare, nei Grigioni, l'engadinese, pare, in un primo tempo, come "lingua dotta" (latina) rifacendosi ad una tradizione letteraria che ci riporta indietro nel tempo fino al 1560 ,

1 Per la fase prescientifica degli studi ladini sono molto utili i lavori di Decurtins 1964 e di J. Kramer che ha studiato (e riedito filologicamente) testi, in particolare la posizione di colui che ci ha fornito le prime notizie sul badiotto, S.P. Bartolomei, nella seconda metà del secolo XVIII; si veda ad es. Le notizie dell'avvocato S.P. Bartolomei (I709-1763) sul ladino dolomitico, "Archivio per l'Alto Adige" LXXII (1978), pp. 133-159. 
cioè al Nuovo Testamento tradotto dal Bifrun..$^{2}$ Mi pare inutile di ripetere qui che la denominazione "ladino", estesa ad un gruppo di favelle assai vasto e lontane tra di loro, disposte in senso orizzontale, non è di certo indovinata qualora si osservi che essa include anche il Friuli e un' area svizzera, il soprasilvano, come del resto ha osservato anche A. Decurtins 1964, 256--304 specie 273. Come annota il Tagliavini 1972, 377-8, il termine è equivoco poiché con "ladino" si indicava, da tempo, la parlata giudeo-spagnola (o portoghese) dei Balcani e, aggiungiamo noi, che con $a l$ latịni al-Afriqi, nell'arabo di Edrisi, si intende il (neo)latino nord-africano. ${ }^{3}$ Qui non mi soffermo a criticare nuovamente l'altra designazione, assai più fortunata, di "retoromanzo", particolarmente infelice dato che i Reti, di cui sappiamo assai poco, ${ }^{4}$, non sono di certo responsabili delle caratteristiche linguistiche dell'ampia e assai variegata serie di idiomi cui si attribuisce una matrice "retica". ${ }^{5}$ Desideravo invece presentare e perfezionare alcune osservazioni a proposito della concezione ascoliana di "ladino" che non sembra soltanto nominalistica e che ingenera, secondo noi, una grande confusione soprattuto in chi non è esperto della problematica linguistica "retoromanza". Ritengo da tempo che chi affronta tali temi dialettologici, non debba soltanto conoscere minuziosamente le questioni di ordine linguistico, ma debba essere ugualmente esperto in tutti i particolari storici in senso lato (i quali includono pertanto anche conoscenze archeologiche e di epigrafia preromana e romana ecc.) per non dire che i nostri dibattiti, come si vede specie negli ultimi tempi, si concludono più che mai in deduzioni nettamente politiche.

Leggendo e rileggendo i Saggi ladini dell'Ascoli si ha l'impressione che egli considerasse "ladina" di dialetto buona parte dell'Italia settentrionale ed in particolare del Veneto e del Trentino. Ma ciò che determina maggiormente la confusione è l'assenza di qualsiasi supporto di ordine storico (o comunque non si riesce ad intravederlo leggendo le sue fittissime pagine, ricche di dati dialettologici esposti in una forma estremamente sintetica).

Chi ha riassunto il suo pensiero e il suo giudizio sui vari dialetti è ad es. G. Bertoni 1916, 106-113, nel capitolo su i "Dialetti veneti" che si apre con l'ampio paragrafo “Di alcuni contatti tra veneto e ladino“. Rileggendo l'Ascoli e il Bertoni citati si può avere l'impressione che essi alludano a due genti diverse, la ladina e la veneta e che la prima, in certa misura, dominasse in un primo tempo nelle ampie plaghe venete considerate nei Saggi ladini e soprattutto a Venezia, specie in epoca medievale.

2 v. Carlo Battisti, "Il termine "ladino", in Storia linguistica e nazionale delle valli dolomitiche atesine, Firenze 1941, pp. 13-23 (in particolare p. 15).

3 v. T. Lewicki, Une langue romane oubliée de l'Afrique du Nord, in "Rocznik orient." XVII (Cracovia 1958), pp. 415-489, contributo originale che ha peraltro suscitato varie critiche, dato che di codesta lingua si sa, a dir vero, molto poco (tranne la toponomastica).

4 Mi basti di rinviare qui soltanto al mio articolo recente Reti e retico, in L'etrusco e le lingue dell'Italia antica, "Atti del Convegno della Società italiana di Glottologia (Pisa 8-9 dicembre 1984)“, Pisa 1985 , pp. $95-128$.

5 Oltre al totale dissenso dell'Ascoli e alle fondate critiche di Carlo Battisti, espresse in tante sue opere, menziono ad es. Fr. Schürr, Die Alpenromanen, "Vox Romanica" XXII (1963), pp. 100-126 e soprattutto i volumetti miscellanei svizzeri, editi da qualche anno, citati dettagliatamente nel mio articolo alla nota precedente. 
In effetti l'Ascoli ritrova ed elenca i fenomeni linguistici ch'egli ritiene tipici del ladino in numerose sezioni del Trentino e del Veneto. Naturalmente più si va indietro nel tempo e maggiormente affiorano tali tratti nei testi antichi (e di certo essi erano nel parlato in numero assai superiore). Ampio spazio è concesso in prima linea alla sezione ladina tridentina occidentale: Val di Sole (pp. 323-4), Val di Rumo (324-5), Val di Non (soprattutto) 325-332; inoltre al gruppo tridentino orientale e alto-bellunese, e cioè alla Val di Cembra (345), Val di Fiemme (345-349), Val di Fassa (349-353), bacino della Gàdera, cioè Badia e Marebbe (353-361), Val Gardena (361-371), Livinallongo con Colle di S. Lucia (372-375), Rocca d'Agordo cioè R. Pietore (375-377), Cortina d'Ampezzo (377-381), Oltrechiusa Cadorina (381-384), Comèlico (384-388). Dopo la considerazione della posizione di passaggio di Erto e Vajont (388-390), si passa alla ampia Sezione del "Ladino-Veneto", cioè allo studio di quelle aree che hanno perduto parte delle caratteristiche "ladine" e cioè: Val Fiorentina (399-400), Agordino centrale e meridionale (400-402), Val di Zoldo (403) e Cadore centrale (403-406). Seguono poi le varie sezioni che si possono ormai considerare "veneto-ladine" e cioè: "Dal bacino del Sarca al Cismon" (406-410), "Feltre e Belluno" (410-415), "Fra l'alto Bacchiglione e l'alta Livenza" (415-419), "Padova e Verona" (410-433), "L'Istria veneta" (433-447) e finalmente varie pagine sono riservate a "Venezia antica" (448-465) e a "Li(d)o Mazor", strettamente collegato a Venezia antica (465-473). Si potrebbe affermare che la straordinaria trattazione dialettologica dell'Ascoli è in realtà una delle prime e migliori descrizioni del Veneto dialettale con in testa le aree che presentano più netti e bene conservati i tratti definiti ladini. Ma si ha sempre l'impressione che l'Ascoli consideri veneto e ladino due entità etniche(??) separate e che la prima abbia, nel tempo, soffocato ed in parte annullato gli originari caratteri ladini della massima parte della regione.

Non è difficile reinterpretare, secondo una visione corretta, i rapporti considerati dall'Ascoli e, come ho detto, riesposti ad es. dal Bertoni.

Sul problema generale ha discorso anche A. Prati in una appendice ai suoi studi di toponomastica veneta: "Pretesi ladinismi della toponomastica veneta" (Prati 1914, 185-193).

E' ovvio che non sono le genti ladine e venete a compiere degli spostamenti o delle invasioni, ma sono soltanto le isoglosse che si spostano, collegate con la storia, la cultura e il prestigio (dovuto a vari fattori). La facies linguistica dell'Italia nordorientale (dato che qui ci limitiamo a discutere di tale regione) era ben diversa verso il $1000 / 1100$ e agli inizi della Scripta veneta (a volte, in parte, bugiarda) rispetto alle epoche successive e ancor più moderne e contemporanee. Così il vero veneziano delle origini dovette offrirci una favella non tanto dissimile dal veneto di tipo settentrionale (nel quale rientravano i linguaggi di Treviso e soprattutto di Oderzo) ed è assai verosimile - come riconoscono gli storici - che buona parte degli immigrati dalla Terraferma nelle isole della laguna verso i secoli VI/VIII, e dopo, provenissero da Oderzo ed Eraclea (filiazione della notevole cittadina romana di Opitergium, 
quando questa venne distrutta dai Longobardi dapprima nel 643 da Rotari e ancora devastata successivamente da Grimoaldo nel 685). E tale parlata lagunare, in un primo tempo di tipo veneto nettamente settentrionale, contrastava col veneto meridionale che ebbe il suo centro in Padova, poi letterariamente rappresentata dal pavano. E qui potremmo citare a mo' di confronto tra ladino e veneto le vicende dialettologiche proprio di Padova, Rovigo e Vicenza (specie meridionale). Forse che sono mutate le genti nel corso degli ultimi cinque secoli se ora riscontriamo che il dialetto di Padova è profondamente diverso dal pavano di Ruzzante, tanto da risultare quasi incomprensibile agli attuali abitatori della città? Il Prati (op. cit.), dopo aver contestato l'esattezza delle spiegazioni dell'Ascoli (Saggi 464-5), sottolinea la "tendenza a ritenere di origine ladina certe forme venete, trentine" ecc... "si avverte pure in altri studiosi venuti dopo l'Ascoli... i quali non si sono mostrati alieni dallo spiegare qualche nome locale veneto secondo la fonetica ladina ove ora si parlano dialetti veneti cosi diversi e per molti rispetti opposti a quelli“. Queste e altre osservazioni del Prati sono in parte esatte, ma viste in prospettiva diacronica possono definirsi anche errate per un grosso equivoco di non considerare i dialetti secondo varie fasi cronologiche. Secondo noi si esprime assai meglio E. Quaresima 1955, 102, quando osserva che "l'Ascoli definisce insigni caratteri ladini ogni importante fenomeno da lui scoperto presso i parlari ladini, il quale ricorre per caso anche presso qualche varietà dei dialetti veneti. Sembra invece più giusto affermare che tutti quei caratteri che il ladino ha in comune col dialetto veneto sono caratteri veneti; stante che nel caso attuale il datore è sicuramente la popolosa ed industre Regione veneta e non la povera e periferica montagna; e il rapporto inverso non è nemmeno concepibile. Il fatto è che i dialetti ladini sono della stessa natura del dialetto veneto originario...". Anche la osservazione del Q., fondamentalmente esatta, meriterebbe ulteriori precisazioni e qualche chiosa che io ho già formulata altrove, in specie per la classificazione delle parlate venete. ${ }^{6}$ Non mi soffermerò qui a dimostrare o negare i rapporti del ladino atesino (o del Sella) con l'ampia regione friulana, dato che anche l'Ascoli ne aveva compreso le modeste convergenze. Anche di ciò spero di avere scritto in altre occasioni con qualche fondamento di verità e soprattutto recentemente ho potuto rilevare che le premesse della latinità importata sono assai diverse. La latinità aquileiese, $\mathrm{e}$ conseguentemente friulana (con antiche propaggini cadorine) è assai diversa dalla latinità (sostanzialmente cisalpina) che sta alla base delle favelle ladine atesine. Non è una ipotesi tanto fantastica di pensare per la latinità aquileiese, di norma più arcaica, ad origini dall'Italia centrale ed in particolare dalla regione dei Sanniti: ipotesi già proposta dagli storici e da me integrata con qualche corollario lessicale. ${ }^{7}$ Altrimenti come si può spiegare un particolare incontrovertibile, e per me di grande rilevanza, facilmente controllabile, e cioè l'incomprensione da parte dei Friulani degli idiomi ladini del Sella. ${ }^{8}$ Gran parte del lessico friulano diverge da quello del ladino

6 v. il mio volume Saggi sul ladino dolomitico e sul friulano, Bari 1972, ad es. a p. 126, nota.

7 Si veda ora il mio lavoro Le denominazioni del "fulmine" e la "latinità aquileiese" nella "Miscellanea di studi alla memoria di E. Giammarco", Pisa 1990.

8 Non è tanto infrequente il caso che i Friulani si avvalgano dell'interprete per capire i dialetti dolomitici atesini, come è avvenuto di recente in occasione del bimillenario del ladino (??) alla cui celebrazione hanno partecipato anche alcuni Friulani. 
atesino e non gioca qui, secondo noi, un ruolo veramente fondamentale l'abbondanza dei tedeschismi dei dialetti del Sella. Ben diversi sono invece i rapporti linguistici tra friulano, specie carnico, e dialetti cadorini (compresi i comeliani e l'ampezzano), anche se l'antica friulanità — anche dialettale - del Cadore è in buona parte dileguata.

Ma passiamo ora in rassegna le concordanze venete col ladino (o viceversa) come sono esposte dal Bertoni cit. integrate da alcuni dati che si possono desumere direttamente dall'Ascoli 1873. Non mancheremo di aggiornare le nostre conoscenze attuali dei vari dialetti, sia pure con semplici rinvii bibliografici per non ripetere quanto ho già altrove annotato e per non appesantire questo mio intervento, rinviando poi all'articolo che ho sovra annunziato.

a) Quanto scrive il Bertoni (pp. 106-7) a proposito delle concordanze nell'uso di -óm, -ón alla $\mathrm{l}^{\mathrm{a}}$. pl. è forse di scarsa rilevanza. La desinenza -ón è comunque tipica del veneto specie settentrionale e rustico ed era comune anche nel pavano; non mi risulta invece che caratterizzasse il veneziano e nemmeno il veronese; $v$. su tutto ciò Pellegrini 1977, 133-39. b) Non capisco quanto sia tratto di "ladinità" (p. 107) il volgere di -atu ad -ò di cui si hanno vari esempi, come filò (da fi l a t u), tratto oltremodo tipico del pavano. c) Il dittongo di $o$ ed $e$ in posizione è indubbiamente attestato nei dialetti veneti e non soltanto alpini; qui mi basterebbe citare casi della toponomastica quali meza - tiera (toponomastica urbana di Belluno, Feltre e Vittorio Veneto), ven. Valier da V a le ri u ecc., piet(o) < pect us, molto diffuso anche in provincia di Rovigo, daspuò 'dopo' $<$ de post, zuóia $<*^{*}$ ugia, truoi $<$ t $\mathrm{r}$ o g i u m, fuorsi 'forse' (Cavassico), viesti 'vesti', biei, fardiei 'fratelli', piez(o) ecc.; d) Ma di gran lunga più importante per le impronte "ladine" è la conservazione di $-S$ finale latino (pp. 107-108) che nei dialetti caratterizza soprattutto il tipo settentrionale, onde anche il veneziano, specie antico; qui rinvio a Pellegrini 1977 e soprattutto $1987,131-2$ e $1987 \mathrm{a}, 49-61$; oltre alla conservazione nelle forme plurisillabe del verbo alla 2. persona sg., bisognerà tenere in considerazione un caso di plurale sigmatico in una lettera veneziana della metà del sec. XIV (li presis 'i prezzi'). Questo tratto distingue abbastanza nettamente - secondo noi - il veneto settentrionale da quello meridionale con centro nella vecchia e prestigiosa Padova. e) Anche la sincope di $-e$-non è estranea ai dialetti veneti, specie negli infiniti e non soltanto nel veronese (che secondo noi, nella fase antica, è piuttosto un dialetto lombardeggiante), ma anche nel trevisano e bellunese (ad es. estre 'essere'). Nell'Egloga trevisana di Morèl (p. 256) si ha vere 'vedere' da vedre 'vedere', crere 'credere', letran ecc. nel Cavassico 315: letra, zucre, povre, vendre, metre, crere. Ma la rilevanza di tale sincope ai fini dei parametri "ladini“ dell'Ascoli è ancora modesta (non così ad es. per il Gartner); f) Anche per la conservazione dei nessi con $L(P L, F L, B L, C L, G L)$ gli esempi veneti si infittiscono più si va indietro con la lettura dei testi (anche se, a volte, si tratta di latinismi grafici) o ci si rivolge alla periferia. Ma testimonianze preziose ci vengono dalla toponomastica nella pronuncia locale (come Plaf 'Piave' a Vittorio Veneto ecc.). Del resto l'ampia conservazione di $L$ nei suddetti nessi è soprattuto attestata nei dialetti delle province di Sondrio, di Bergamo e di Brescia (mi basti rin- 
viare alla mia Carta dei dialetti d'Italia, Pisa 1977 b); g) Il Bertoni (pp. 108-9), come altri studiosi tra i quali anche il Devoto, erra nel ritenere un tratto "ladino" la presenza delle interdentali sorda e sonora (ẑinkue, žènto o jente 'gente' ecc.). Si tratta infatti di una caratteristica tipicamente veneta ed in origine meridionale con centro verosimile in Padova, diffusa ben presto in tutte le direzioni (ma non a Venezia, secondo noi); essa ha ora una ampia attestazione nella provincia di Treviso (rustica) e nel Bellunese, ivi compreso il Cadore ed il Comèlico (ma essa non ha raggiunto Cortina d'Ampezzo), nell'Agordino sino a San Tommaso, ma non ad Alleghe. h) Poca rilevanza ha pure l'imperfetto in -eva per -ava diffuso nel veneto antico (già nell'Indovinello veronese si ha pareva per parava). Analoga osservazione vale per $-e$, vocale di appoggio ad es. nella prima persona: dighe, fae, mane, parle (p. 109) per $-o$, forme ora tipiche del veneto settentrionale; l) $\mathrm{E}$ ' invece fondamentale per la ladinità la palatalizzazione di $C A$ e $G A$ (così essa è infatti ritenuta tuttora) per la quale l'Ascoli citava per l'area veneta soltanto l'esempio di chian, chiani di Fra Paolino Minorita, De Regimine rectoris (edito dal Mussafia). Giustamente H. Schmid 1956 ha ribadito che si tratta di un fenomeno diffuso un tempo nella Cisalpina, di cui ci rimangono ora soltanto delle tracce (e l'Ascoli lo riteneva il fenomeno più tipico della "ladinità", contrassegnato dalla lettera $\alpha$ ). Gli esempi di codesto fenomeno, evidentemente non bene esplorato nel secolo passato e nel nostro, stanno moltiplicandosi tanto per la documentazione di appellativi, quanto per le tracce toponimiche assai numerose; rinvio intanto solo a Pellegrini 1977, 315, Tomasi-Pellegrini 1983, Vigolo 1987 e 1989 e dovrei aggiungere altri importanti esempi che mi sono stati segnalati, da non molto tempo, per l'area trevisana settentrionale e per l'Agordino centrale. Anche secondo la nostra visuale tale palatalizzazione dovrebbe essere considerata un tratto arcaico della fonetica cisalpina e del veneto (forse bisognerà rivedere, a questo proposito, anche i modi di costituzione del franco-veneto, forse motivo di concordanza - come tanti altri - tra cisalpino e veneto e francese antico). A questo punto conviene forse citare le parole del Bertoni (p. 109): "Più risaliamo a ritroso la corrente dei secoli, e maggiormente salde si fanno le attestazioni fra ladino e veneto“ e poi il Bertoni prosegue quasi con le parole dell'Ascoli 1873 464-65 (che, a dir vero, hanno una modesta rilevanza): “E' questa una constatazione, la quale non manca di gettare qualche luce sulle origini di Venezia, in quanto rischiara un poco la via che i documenti storici tracciano infida alla migrazione dalla terraferma alla laguna. Venezia fu ladina [??! quale equivoco!] se anche oggi sono venute a perdersi le caratteristiche ladine, come ladina fu Trieste, che fini con l'accogliere il dialetto soggiacendo all'impero linguistico della regina dell'Adriatico“ (ma, a dir vero, Trieste fu friulana, non ladina!). L'Ascoli 1973, 337 accenna ad altri "caratteri fondamentali del sistema fonetico ladino" ed oltre a quelli già citati, mi pare opportuno menzionare: la formazione di dittonghi discendenti da $e$ stretto $(e, i)$ e da $o$ stretto $(o, u)$ di cui si hanno ampie attestazioni nel Veneto settentrionale e soprattutto nei nomi locali; oltre agli esempi da me raccolti nell'Alpago (BL), v. Pellegrini 1987, 363-4, si dovrà tenere in considerazione l'ampia raccolta bellunese e alto-trevisana riunita da G. Tomasi 1988, 316-7; si notino toponimi quali Carpenéi, Pinéi, Salzéi, Borséi, Spinéi, Roréi, Cornéi ecc. (Alpago), Nogaréi, Spinéi, Lastéi, Salcéi, Roréi, (Frusseda, cioè Ponte nelle Alpi), Lastéi, Arnéi, Tasséi, Zoppéi, 
Spinéi, Corneléi, Landréi (Castion di Belluno), Nespoléi (Revine), Roréi, Favéi, Alnéi (Trichiana), Roréi (Lentiai), Zopéi (Vittorio Veneto), Gatéi, Canapéi (Lago), Salazéi, Curtoléi (Miane), Faveréi (Credazzo), ecc.

Ed infine tra i tratti fonetici del "ladino" non si dimenticherà il fenomeno dei dittonghi induriti (verhärtete Diphtonge) ai quali molti linguisti, a partire da Th. Gartner, attribuiscono tanta specificità anche per i rapporti tra "retoromanzo" e franco-provenzale. Essi sono comuni nel grigionese e non sono assenti in alcuni dialetti friulani, mentre mancano quasi del tutto nei dialetti ladini atesini. Qui bisogna invece sottolineare che essi sono frequenti nel bellunese antico (Cavassico) e nella toponomastica agordina (Pellegrini 1955, 24 e 31-32). Ora il Tomasi 1988, 317 cita esempi assai interessanti per l'alto trevigiano tra cui sék da precedente séi $<$ s i i (m) 'sete' (Falzé di Piave) e vari casi della toponomastica tra cui Pianéch (Castion), Lastréch (Lago), forse Castelic (Tarzo) ed altri ancora (comunicatimi a voce).

Ma è fondamentale lo studio delle aree lessicali di alcuni termini che sono ritenuti spesso caratterizzanti del "retoromanzo" (e sarebbero spesso esclusivi del "ladino"). Su tale problema ho già dissertato più volte e non intendo qui ripetermi anche se i miei rilievi - inequivocabili - non hanno trovato alcuna valorizzazione, né attenzione (v. ad es. Pellegrini 1986). E qui non starò a ripetere alcune critiche a proposito di "lessico retoromanzo" già rivolte correttamente al Gartner $1883 \mathrm{da}$ parte di C. Battisti 1937. Sarebbe invece opportuno accennare, sempre a proposito del medesimo tema, alla disinformazione lessicale di H. Kuen 1937, il quale tenta pure di identificare una serie di voci che sarebbero tipiche ed esclusive dell'area sunnominata. Così ad es. il $\mathrm{K}$. cita come tipici del retoromanzo termini quali e go per mihi $(>m i)$, soror, frater, he bd o ma, *l is u ra che non è una parola sia pure ricostruita, ma soltanto una "tipizzazione", $9 *^{*} \mathrm{k}$ aitho 'cucchiaio', a bu nde 'abbastanza“, a qa le 'solco d'acqua', gr a nit t a 'mirtillo rosso': tutte voci delle quali credo di aver illustrato l'area assai più correttamente in tanti (e troppi!) scritti; così dicasi altrettanto per de-avorsu, ${ }^{*}$ nullia, acutus, a cidu s, *dragiu, cella riu, bà pt is mu per baptisimus (503) ${ }^{10}$ ecc.; si veda ora il mio articolo Ladinisch: Interne Sprachgeschichte II. Lexik/Evoluzione del lessico, in LRL III (Tübingen 1989), pp. 667-679. A proposito di "lessico retoromanzo“ è assai curiosa la chiosa del Meyer-Lübke, REW 8934 *troju 'Fussweg', 'Steig', 'Holzschleife': 'Das spezifisch rätorom. Wort ist wohl vorröm."; credo sia ben noto agli studiosi quale è l'area di troj, trodzo (e varianti) che comprende la massima parte del veneto (anche meridionale), il Trentino e parte della Cisalpina. Non so proprio come essa possa dichiararsi "specifica del 'retoromanzo'“.

9 v. il mio articolo Un continuatore di lu de r e: *l u s u ra 'articolazione', in "Studi mediolatini e volgari“ XXVIII (1981), pp. 59-66.

10 I miei articoli etimologici sul ladino e friulano (ed in generale sull'Italia nord-orientale) sono elencati nella Bibliografia degli scritti linguistici di G.B.P. (1945-1987), Padova (Dipartimento di linguistica del'Università) 1987, con indici. Ora dovrei aggiungere una nuova ottantina di contributi. 
Purtroppo sul "retoromanzo" o ladino gravano ancora tanti pregiudizi ed è assai difficile convincere gli studiosi sulla reale situazione dei rapporti linguistici e sulle autentiche relazioni: esse non si svolgono affatto in senso orizzontale, secondo la tradizione petrificata, ma assai chiaramente, e logicamente, in senso verticale, contrariamente a quanto si legge nei manuali di linguistica romanza; questi, volutamente o per ignoranza, non si peritano di citare tesi antitradizionali e che in sostanza non sono mai state demolite (alludo ad es. agli insegnamenti di Carlo Battisti); tesi che sono state sviscerate da ricerche puntuali durate per oltre un settantennio. Qui ho voluto soltanto anticipare brevemente alcuni concetti che saranno invece trattati diffusamente nel contributo qui sopra annunciato.

\section{ABBREVIAZIONI BIBLIOGRAFICHE}

Ascoli 1873 = G.I. Ascoli, Saggi ladini (= AGI I).

Battisti $1937=$ C. Battisti, Storia della questione ladina, Firenze 1937.

Bertoni 1916 = G. Bertoni, L'Italia dialettale, Milano 1916.

Decurtins 1964 = A. Decurtins, Das Rätoromanische und die Sprachforschung, "Vox Romanica“ XXIII/2 (1964), pp. 256-304.

Gartner 1883 = Th. Gartner, Rätoromanische Grammatik, Heilbronn 1883.

Kuen $1937=\mathrm{H}$. Kuen, Die ladinischen Dolomitenmundarten in der Forschung der letzten zehn Jahre (1925-1935), "Zeitschrift für romanische Philologie“ LVII (1937), pp. 481-520.

Pellegrini 1955 = G.B. Pellegrini, Schizzo fonetico dei dialetti agordini, Venezia 1955.

Pellegrini 1977 = G.B. Pellegrini, Studi di dialettologia e filologia veneta, Pisa 1977.

Pellegrini 1977a = G.B. Pellegrini, Carta dei dialetti d'Italia con un volumetto di commento, Pisa 1977.

Pellegrini 1987 = G.B. Pellegrini, Ricerche di toponomastica veneta, Padova 1987.

Pellegrini 1987a = G.B. Pellegrini, Quarant'anni di ricerche sul "retoromanzo" in "Rätoromanisch heute", herausgg. von G. Holtus und J. Kramer, Tübingen 1987, pp. 49-61.

Pellegrini 1986 = G.B. Pellegrini, Considerazioni sui rapporti lessicali del fassano, "Mondo ladino" X(1986), pp. 359-373.

Prati 1914 = A. Prati, Escursioni toponomastiche nel Veneto II, RDR VI (1914), pp. 139-194.

Quaresima 1955 E. Quaresima, Parentela veneto-ladina, Trento 1955.

Tagliavini $1972=\mathrm{C}$. Tagliavini, Le origini delle lingue neolatine, ${ }^{6}$ Bologna 1972.

Schmid $1956=\mathrm{H}$. Schmid, Ueber die Palatalisierung von C, G vor A im Romanischen, "Vox Romanica" XV(1956), pp. 53-80.

Tomasi-Pellegrini 1983 = G. Tomasi, Dizionario del dialetto di Revine (TV), con Prefazione di G.B. Pellegrini, Belluno 1983.

Tomasi 1988 = G. Tomasi, Note di toponomastica prealpina, "Archivio per l'Alto Adige“ LXXXII(1988), pp. 295-317. 
Vigolo $1987=\mathrm{T}$. Vigolo, La palatalizzazione di $C, G+A$ nei dialetti veneti, “AGI"LXXI fasc. 1-2 (1986), pp. 60-80.

Vigolo 1989 = T. Vigolo, La palatalizzazione di CA e GA nel dialetto vicentino, in Quaderni patavini di linguistica, Monografie 6. pp. 383-389.

Povzetek

\section{NEKAJ OPAZK K “RETOROMANSČINI"}

Avtor je ognjevit pobornik ideje o jezikovni nepovezanosti romanskih alpskih govorov (Furlanija, Dolomiti, romanski Graubünden), ki jih večina učbenikov romanskega jezikoslovja uvršča v retoromansko ali, $z \vee$ italijanski lingvistiki rabljenim izrazom, ladinsko jezikovno skupino, (gl. njegovo izčrpno studijo Ling. XXII, 1982, str. 3-64). Tópot predstavlja svoje poglede v zelo zgoščeni obliki. Kritično pretresa predvsem ideje začetnika teorije o povezanosti teh romanskih področij, vélikega italijanskega jezikoslovca G.I. Ascolija, pa tudi avstrijskih romanistov Th. Gartnerja in H. Kuhna. Dokazuje, da ni nobenega jezikovnega pojava, ki bi bil izrazito ladinski, torej neznan kateremu drugemu romanskemu govoru. Tako so, npr., ohranjanje soglasniških skupin z likvido kot PL-, FL-, ohranjanje končnega $-s$, sinkopa, ali pa znamenita alpska palatalizacija velarov v sekvenci CA-, GA znani tudi posameznim beneškim govorom. Avtor ravnotako zanika, da bi v alpskih govorih bili izrazi, torei izrazito retoromanski ali ladinski izrazi, ki bi drugod ne bili poznani. Opozarja na zmotno Ascolijevo stališče, da namreč gre za dve različni etnični plasti, ladinsko in beneško; in ta naj bi bila jezikovno obvladala prvo. Gre le za izoglose, katerim pogojuje razsežnost jezikovni prestiž nekega govora. 